\title{
Distribution des vitesses et des concentrations dans un écoulement diphasique liquide/solide à surface libre
}

\author{
Velocity and concentration distributions in liquid/solid \\ two-phase free-surface flow
}

\author{
Y. Hassanzadeh \\ laboratoire de Mécanique Expérimentale des Fluides, \\ IMTA Université Pierre et Marie Curie \\ Campus d'Orsay 91405
}

\section{Distribution des vitesses}

\section{Introduction}

Dans le cas d'un écoulement plan turbulent monophasique, dans la zone près d'une paroi rigide, les théories de longueur de mélange de Prandtl et de similitude de Von Karman conduisent à la loi de distribution logarithmique des vitesses. La validité de cette loi a été vérifiée par $\mathrm{J}$. Nikuradse dans le cas d'un écoulement turbulent monophasique, dans une conduite circulaire, dans le cas où les parois étaient hydrauliquement lisses et rugueuses. G.H. Keulegan a montré par la suite que la loi logarithmique des vitesses locales était également valable pour des écoulements monophasiques rectilignes uniformes à surface libre. La question qui s'est posée très vite était de savoir si la distribution des vitesses locales était également logarithmique dans le cas des écoulements diphasiques liquide-solide dans des canaux à surface libre. Pour répondre à cette question, plusieurs auteurs se sont intéressés à la recherche en nature et sur les modèles réduits. Parmi eux, on peut citer les travaux de : V.A. Vanoni [2] (1946) ; E. Meyer-Peter et R. Muller [3] (1948); H.A. Einstein et Ning-Chien [4] (1955) et R.J. Garde et A.S. Paintal [5] (1964).

L'ensemble de ces recherches a mis en évidence que, d'une part la loi logarithmique des vitesses est valable, dans un écoulement chargé de granulats en suspension, et, d'autre part, que la constante de Karman $K$ n'est pas une véritable constante ; elle varie de façon appréciable. Mais à notre connaissance, la variation des constantes $B$ et $C$ de l'équation de distribution des vitesses (voir plus loin) avec la concentration et la dimension des particules solides n'est pas illustrée.

\section{Installation expérimentale}

Le schéma de l'installation d'essais est montré sur la figure 1 . Le circuit de l'écoulement étant fermé, le régime est donc permanent en moyenne. Les essais ont été réalisés dans un canal vitré de section rectangulaire de $35 \mathrm{~cm}$ de largeur, $25 \mathrm{~cm}$ de hauteur, et $18 \mathrm{~m}$

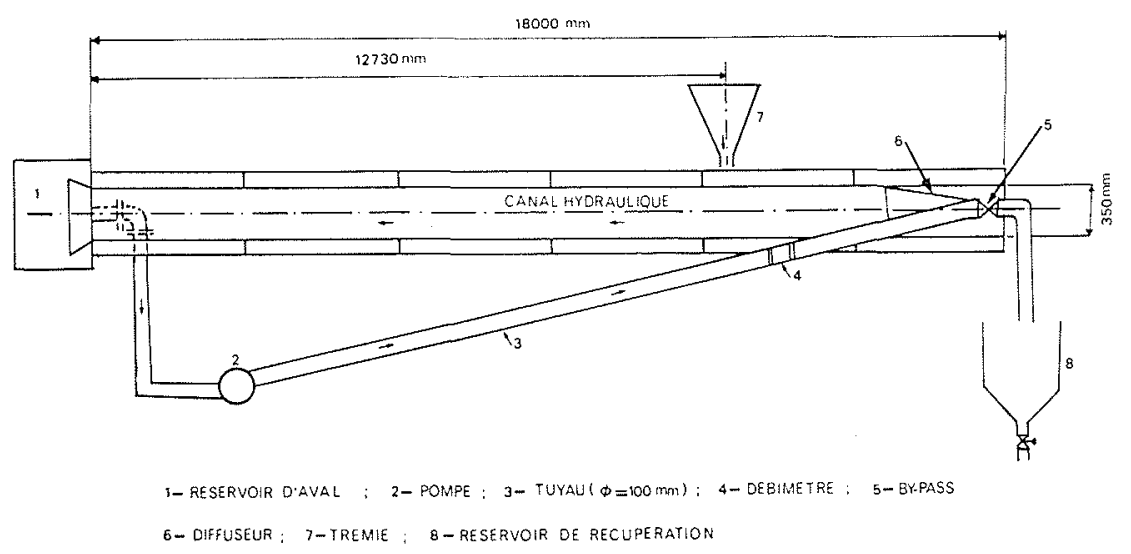

Figure 1 - Schéma de l'installation - "circuit fermé". 


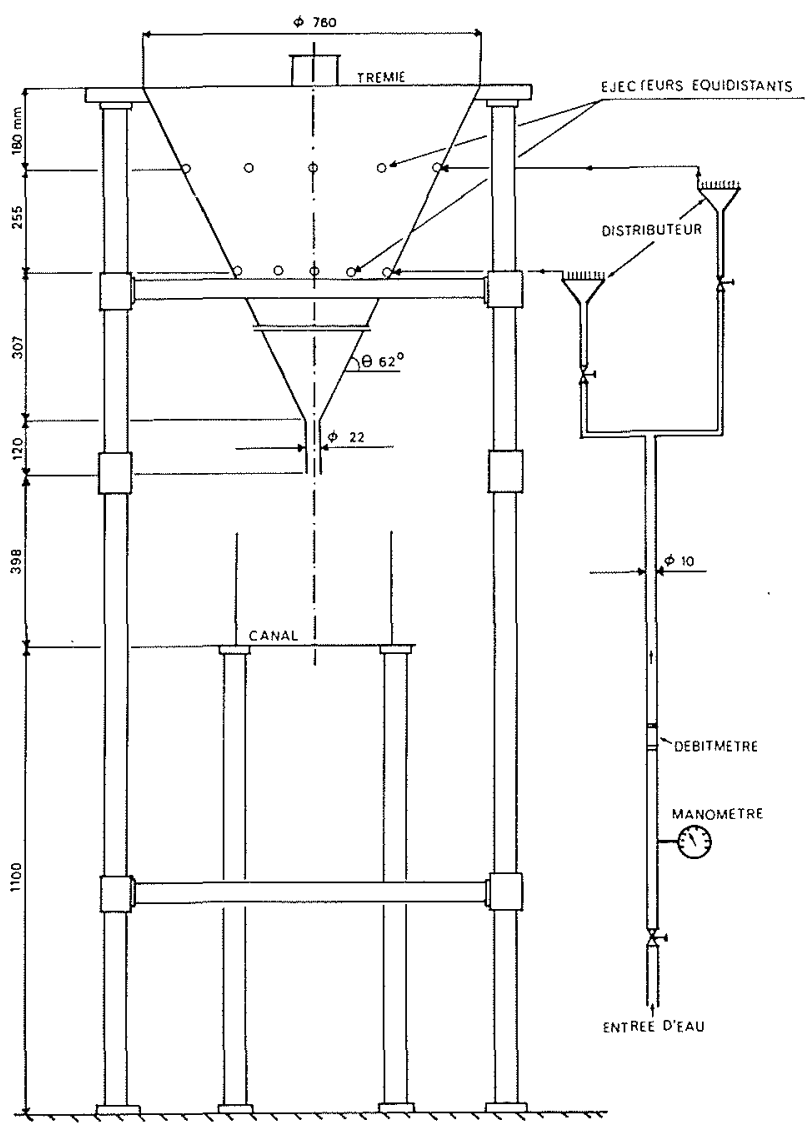

Figure 2 - Installation d'introduction des particules solides.

de longueur. La pente du canal est réglable, et, dans nos expériences, elle est égale à $I=0,00733$. Les parois du canal sont en verre de sécurité de $7 \mathrm{~mm}$ d'épaisseur, et elles sont parfaitement lisses dans le cas des écoulements monophasiques. On introduit le liquide dans le circuit à partir du réservoir d'aval, on remplit le réservoir d'aval, et on fait fonctionner la pompe. A l'aide du système de by-pass, on ferme parfaitement la vanne de vidange et on ouvre lentement la vanne d'entrée du canal. Le fluide, passant dans la pompe, s'écoule alors dans le tuyau avec une vitesse débitante de l'ordre de $3,4 \mathrm{~m} / \mathrm{s}$ pour un débit du fluide estimé à $27 \mathrm{l} / \mathrm{s}$. A la fin du tuyau, un diffuseur permet de réduire la vitesse à l'entrée du canal et on met une grille à un mètre de la fin du diffuseur, afin de minimiser la perturbation provoquée par la pompe. L'introduction des particules solides se fait par un dispositif simple, figure 2 , qui injecte le mélange liquide-solide avec un débit de l'ordre de $0,125 \mathrm{l} / \mathrm{s}$, au début de chaque expérience, au milieu du canal, pendant un intervalle de temps $t_{p}$ égal au temps moyen de parcours d'un élément de volume le long du circuit fermé. D'une façon générale, pour homogéniser l'écoulement, initialement, nous avons tenu compte que le temps d'introduction $t_{i}$ des particules solides, pour un écoulement diphasique liquidesolide, dans un circuit fermé, devrait être calculé par la relation suivante :

$$
t_{i}=K t_{p} \text { avec } K=1,2,3 \ldots
$$

Afin d'assurer l'homogénéité et l'établissement du régime d'écoulement, après avoir introduit des particules, on laisse recycler l'écoulement plusieurs fois.

\section{Etude expérimentale}

En écoulement diphasique liquide-solide, nous avons effectué 3 séries d'essais ; la première série en présence des particules solides de diamètre moyen $\bar{d}=30 \mu$. Dans la seconde série ce diamètre moyen était de $\bar{d}=$ $40,8 \mu$ et dans la troisième série il était de $\bar{d}=52 \mu$. Notons que les particules solides utilisées dans chaque série étaient des microbilles de verre, de masse volumique $\rho_{s}=2,45 \mathrm{gr} / \mathrm{cm}^{3}$ ayant des formes sphériques et uniformes. La mesure du débit liquide $Q_{L}$ s'effectue à l'aide d'une tuyère. Le tirant d'eau $h$ a été mesuré par l'intermédiaire des limnimètres réglables situés au milieu du canal. Les lectures sont faites à $0,1 \mathrm{~mm}$ près, au vernier. Les prélèvements de 3 limnimètres ont servi pour déterminer le tirant d'eau $h$. En raison des frottements, la température de l'écoulement augmente, ce qui influence la viscosité du fluide. Nous avons mesuré la température de l'écoulement par l'intermédiaire d'un thermomètre localisé dans le réservoir d'aval. Plusieurs lectures ont été faites pendant la réalisation de l'expérience. La valeur moyenne des différents prélèvements est considérée comme la température moyenne de l'écoulement. Dans le tableau 1 , nous donnerons les conditions hydrauliques des expé-

\begin{tabular}{|c|c|c|c|c|c|c|c|c|c|c|c|c|c|}
\hline \multicolumn{14}{|c|}{ Tableau } \\
\hline $\begin{array}{l}N^{\circ} \text { de } \\
\text { série }\end{array}$ & $\begin{array}{l}\bar{d} \\
(w)\end{array}$ & $\begin{array}{l}Q \\
(\Omega / s)\end{array}$ & $\begin{array}{c}Q_{s} \\
(g r / s)\end{array}$ & $\begin{array}{l}{ }^{c}{ }_{T} \\
(\%)\end{array}$ & $\begin{array}{c}\mathrm{h} \\
(\mathrm{cm})\end{array}$ & $\begin{array}{c}\tilde{\gamma} \\
(\mathrm{cm} / \mathrm{s})\end{array}$ & $\begin{array}{c}\mathrm{R}_{\mathrm{H}} \\
(\mathrm{cm})\end{array}$ & $\begin{array}{c}\tilde{u}_{*} \\
(\mathrm{~cm} / \mathrm{s})\end{array}$ & $\begin{array}{c}\tau_{0} \\
\left(\text { dyne } / \mathrm{cm}^{2}\right)\end{array}$ & $\left({ }^{\circ} \mathrm{C}\right)$ & $\begin{array}{c}v \\
(s t)\end{array}$ & ${ }^{F_{r}}$ & $\operatorname{Re}$ \\
\hline eau pure & - & 27,128 & 0 & 0 & 6,28 & 123,42 & 4,62 & 5,76 & 33,18 & 25 & 0,009 & 1,57 & 253425 \\
\hline \multirow{2}{*}{$I$} & \multirow{2}{*}{30} & 27,322 & 103,332 & 0,3782 & 6,28 & 124,30 & 4,62 & 5,76 & 33,18 & 25 & 0,009 & 1,58 & 255348 \\
\hline & & 27,322 & 195,024 & 0,7138 & 6,28 & 124,30 & 4,62 & 5,76 & 33,18 & 25 & 0,009 & 1,58 & 255348 \\
\hline \multirow{2}{*}{ I I } & \multirow{2}{*}{40,8} & 27,129 & 103,307 & 0,3808 & 6,20 & 125,02 & 4,58 & 5,74 & 32,95 & 25 & 0,009 & 1,60 & 254374 \\
\hline & & 27,20 & 217,232 & 0,8494 & 6,22 & 124,94 & 4,59 & 5,75 & 33,06 & 28 & 0,008375 & 1,60 & 273905 \\
\hline \multirow{2}{*}{ III } & \multirow{2}{*}{52} & 27,37 & 79,841 & 0,2723 & 6,21 & 125,93 & 4,58 & 5,74 & 32,95 & 25 & 0,009 & 1,61 & 256329 \\
\hline & & 26,784 & 192,020 & 0,7334 & 6,12 & 125,04 & 4,53 & 5,71 & 32,60 & 27 & 0,0085 & 1,61 & 266800 \\
\hline
\end{tabular}




\begin{tabular}{|c|c|c|c|c|c|c|c|c|c|}
\hline \multicolumn{10}{|c|}{ Tableau 2} \\
\hline \multirow{2}{*}{ Sërie } & $\overline{\mathrm{d}}$ & & A & $A^{\prime}$ & k & $K^{\prime}$ & \multirow{2}{*}{ B } & \multirow{2}{*}{ c } & \multirow{2}{*}{$B^{\prime}$} \\
\hline & (H) & $(\%)$ & P.P( 1$)$ & $L, P(2)$ & $P \cdot P(1)$ & $L \cdot P(2)$ & & & \\
\hline eau pure & - & 0 & 5,695 & 6,616 & 0,404 & 0,348 & 5,126 & 0,193 & 26,237 \\
\hline \multirow{2}{*}{ I } & \multirow{2}{*}{30} & 0,3782 & 5,473 & 6,243 & 0,420 & 0,368 & 5,895 & - & 26,193 \\
\hline & & 0,7138 & 5,322 & 6,646 & 0,432 & 0,346 & 6,683 & $-0,176$ & 26,787 \\
\hline \multirow{2}{*}{ II } & \multirow{2}{*}{40,8} & 0,3808 & 5,332 & 6,814 & 0,431 & 0,338 & 6,206 & - & 26,540 \\
\hline & & 0,8494 & 5,291 & 6,842 & 0,435 & 0,336 & 6,483 & $-0,550$ & 26,800 \\
\hline \multirow{2}{*}{ III } & \multirow{2}{*}{52} & 0,2723 & 5,189 & 6,465 & 0,443 & 0,356 & 6,931 & - & $26,64 \mathrm{~s}$ \\
\hline & & 0,7334 & 4,881 & 6,808 & 0,471 & 0,328 & 8,004 & $-0,638$ & 26,979 \\
\hline (1) P.P & in & se $p$ & de la & paroi & & & & & \\
\hline (2) L. I & ind & ique $10 i$ & $n$ de $1 a$ & paroi & & & & & \\
\hline
\end{tabular}

riences réalisées. La mesure de distribution des vitesses locales dans les écoulements mono et diphasiques a été effectuée au milieu du canal à l'aide d'un tube de pitot. En chaque point nous avons pris 10 à 20 mesures consécutives avec un intervalle de temps $t_{p} \simeq 25$ secondes ; $t_{p}$ désignant le temps moyen de parcours d'un élément de volume le long du circuit fermé. La lecture de pression totale a été faite sur le manomètre à eau à $0,1 \mathrm{~mm}$ près. La précision sur la valeur de la vitesse locale dans le cas de l'écoulement monophasique est trè̀ satisfaisante, car l'erreur relative est inférieure à $0,5 \%$, tandis que la présence de plusieurs paramètres dans la formule de vitesse de l'écoulement diphasique [8] augmente la probabilité d'erreur. En général, pour la vitesse locale de la suspension, nous estimons une erreur relative maximale de l'ordre de $1 \%$.

\section{Résultats concernant la distribution des vitesses}

Nous avons présenté la loi de variation de $\bar{u}$ en fonction de $y$, sous la forme adimensionnelle, de la façon suivante :

- Dans la zone près de la paroi $(y / h<0,2)$ on a :

$$
\bar{u} / u_{*}=A \log \left(u_{*} y / \nu\right)+B
$$

ou

$$
(\bar{u}-U) / u_{*}=A \log y / h+C
$$

- Dans la zone loin de la paroi $(y / h>0,2)$ on a :

$$
\bar{u} / u_{*}=A^{\prime} \operatorname{lig} y / h+B^{\prime}
$$

où $u_{*}$ désigne la vitesse de frottement moyenne, calculée par la relation :

$$
\widetilde{u}_{*}=\sqrt{\tau_{0} / \rho_{L}}=\sqrt{R_{H} I g}
$$

La valeur de $\widetilde{u}_{*}=5,76 \mathrm{~cm} / \mathrm{s}$ trouvée pour l'écoulement monophasique est légèrement inférieure à celle de $u_{*}$ locale calculée par la formule du coefficient de perte de charge $\lambda$ appliquée à la section rectangulaire allongée, sur la paroi lisse :

$$
\begin{gathered}
1 / \sqrt{\lambda}=2 \log R_{e} \sqrt{\lambda}-1,16 \\
v / u_{*}=\sqrt{8 / \lambda}
\end{gathered}
$$

d'où $u_{*}=5,86 \mathrm{~cm} / \mathrm{s}$

Ce résultat est tout à fait normal, car $u_{*}$ est plus petit près des angles. Dans la zone près de la paroi, nous avons trouvé le profil logarithmique des vitesses suivant, pour l'écoulement monophasique :

$$
\bar{u} / \widetilde{u}_{*}=5,695 \log \left(y \cdot \tilde{u}_{*} / \nu\right)+5,126
$$

En introduisant la vitesse de frottement locale $u_{*}$ au lieu de $\widetilde{u}_{*}$, nous obtenons :

$$
\bar{u} / u_{*}=5,598 \log \left(y u_{*} / \nu\right)+4,997
$$

Nous avons porté sur le tableau 2, les valeurs obtenues pour $A, B, C, K, A^{\prime}, B^{\prime}, K^{\prime}$ en fonction des différentes concentrations et des différentes séries de particules solides.

\section{Distribution des concentrations}

\section{Introduction}

Pour donner les variations de la concentration des granulats en suspension, on part de l'équation différentielle donné par M. P. O’Brien [9] (1933):

$$
\bar{c} V_{c}+D_{s} \frac{d \bar{c}}{d y}=0
$$

H. Rouse [10] (1938) suppose que $D_{s}=D_{L}$, et, en prenant la distribution linéaire des forces de forttement et la dérivée de la loi de distribution des vitesses déficitaires, il aboutit à :

$$
D_{L}=k u_{*}(1-y / h) y
$$




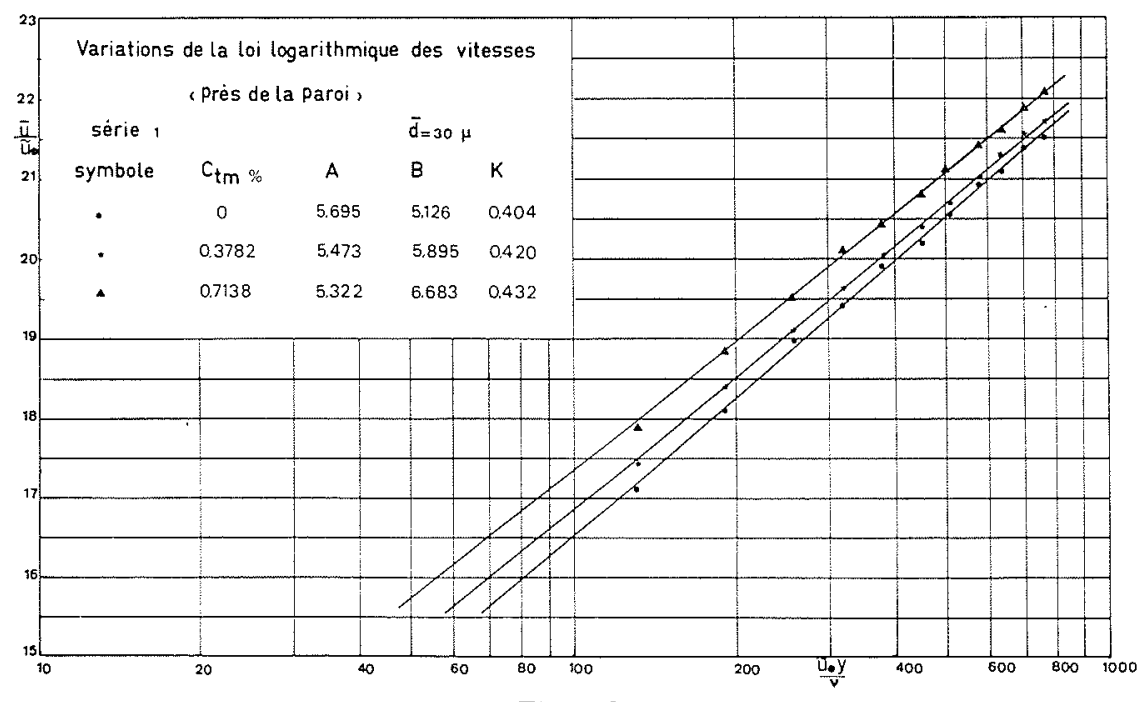

Figure 3
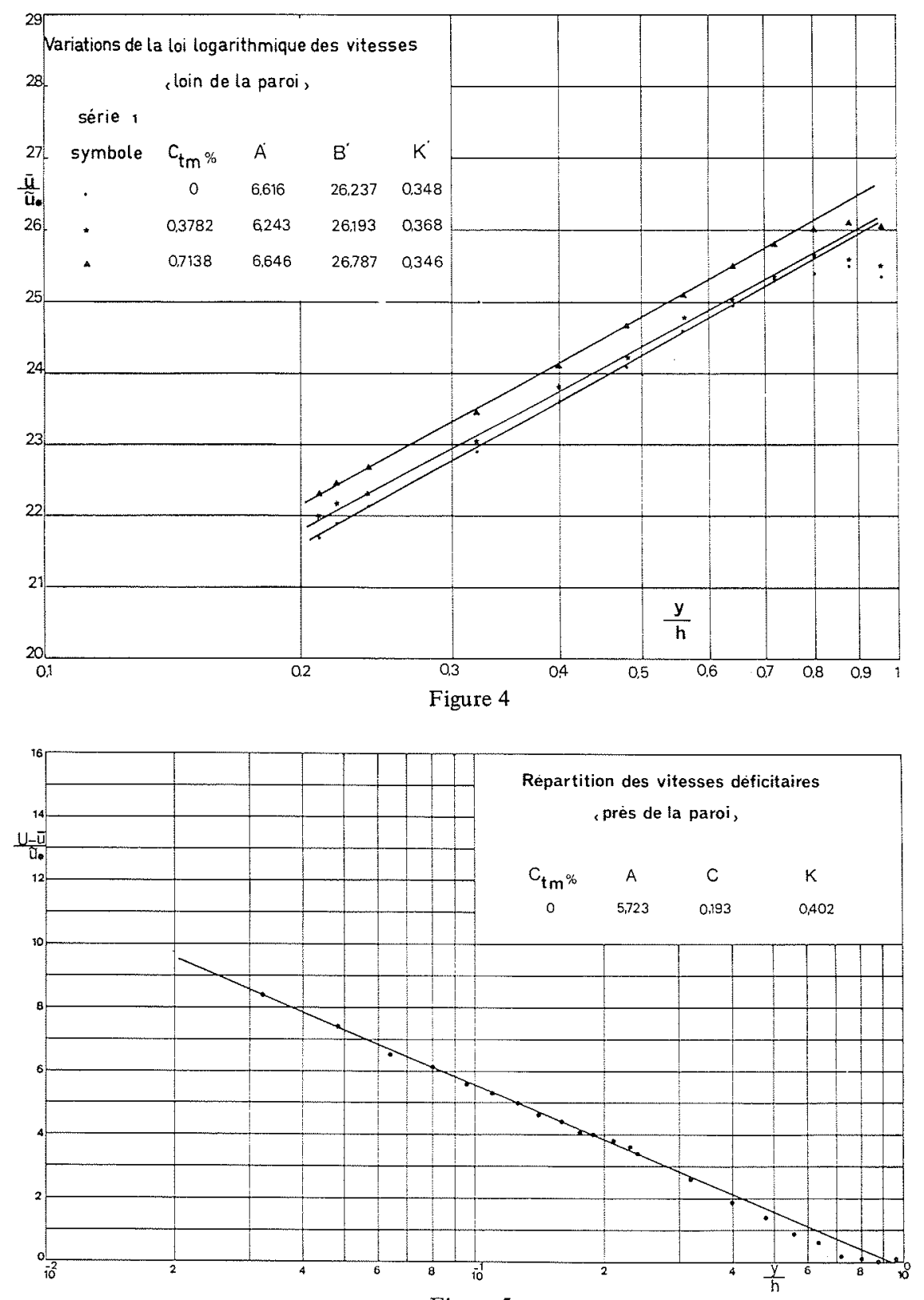

Figure 5 


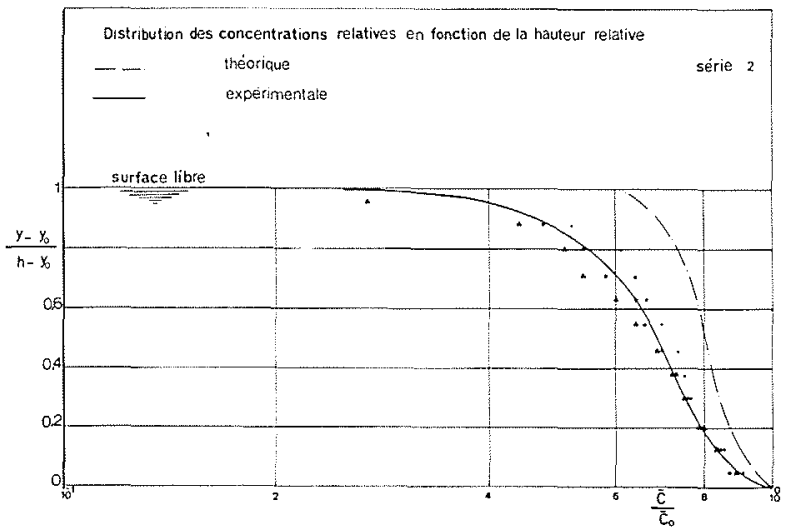

Figure 6

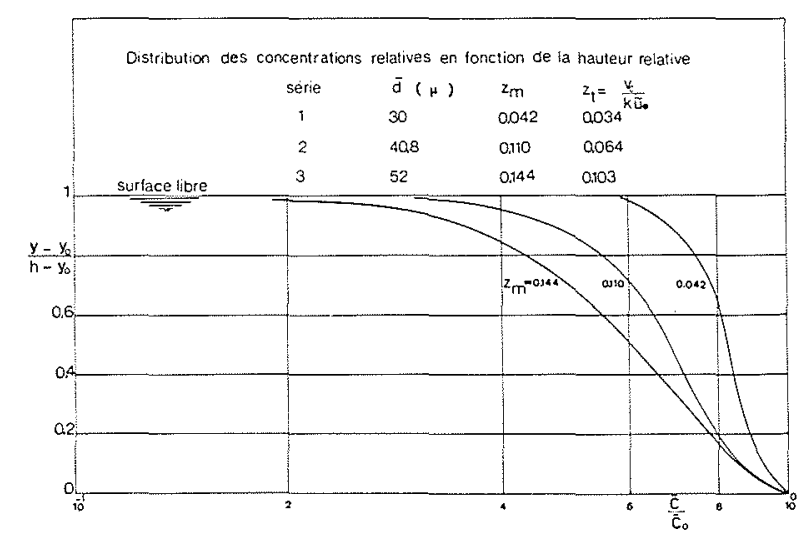

Figure 8

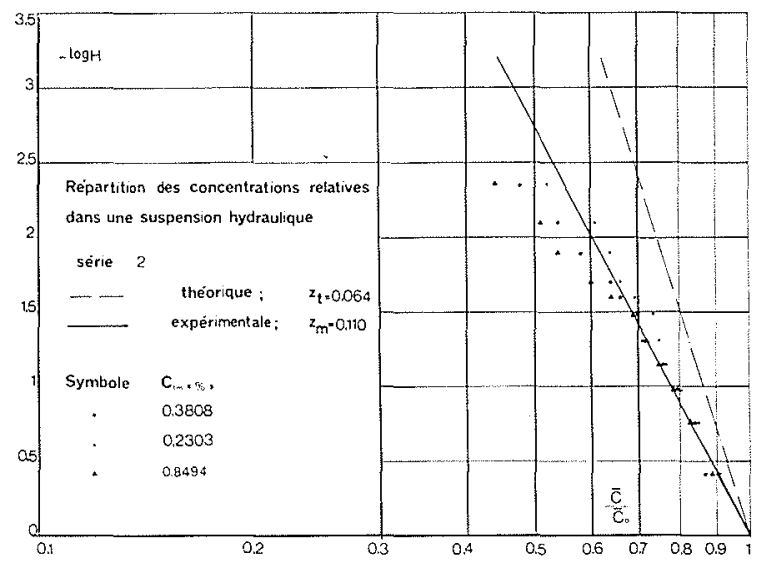

Figure 7

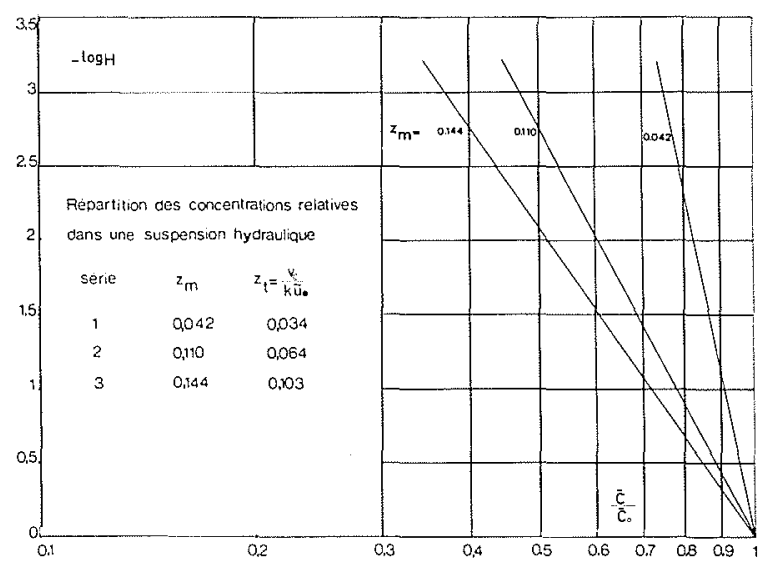

Figure 9

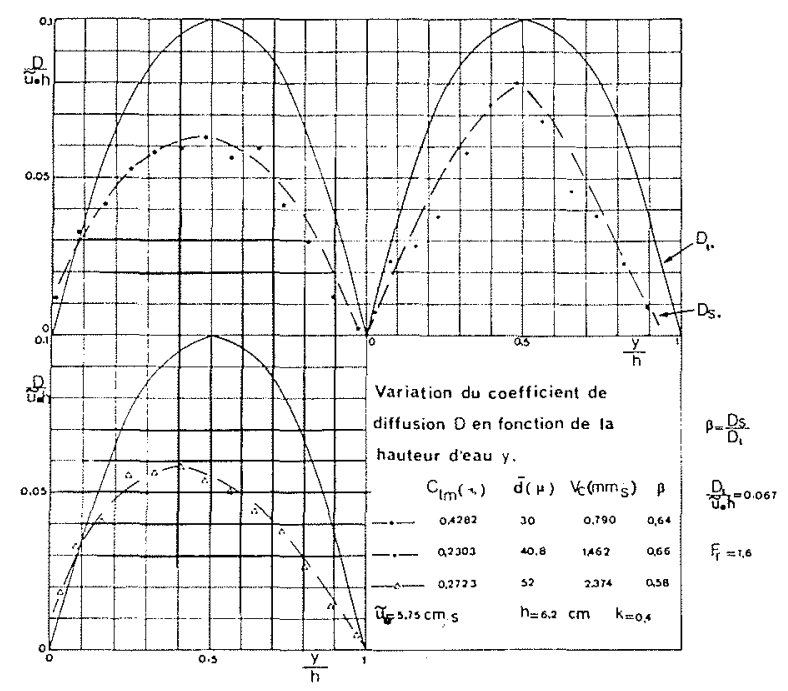

Figure 10 
Compte tenu de ces deux équations, Rouse a déduit la relation suivante :

$$
\bar{c} / \bar{c}_{0}=\left[\frac{h-y}{y} \frac{y_{0}}{h-y_{0}}\right]^{\frac{V_{c}}{K u *}}=H^{Z}
$$

ou

$$
\log \bar{c} / \bar{c}_{0}=Z \log H
$$

ou

$$
V_{c}=\frac{1}{18}\left(\rho_{s} / \rho_{L}-1\right) \bar{d}^{2} g / \nu \quad ; \quad \tilde{u}_{*}=\sqrt{R_{H} I^{g}}
$$

La vérification expérimentale de ces équations a été faite par plusieurs chercheurs ; parmi eux, nous rappelons les résultats de : V.A. Vanoni [2] (1941) ; H.A. Einstein et Ning-Chien [4] (1954) ; R. Durand [6] (1951) et M. Abourayan [7] (1974). Mais il faut mettre un exposant $Z_{m}$ mesuré, différent de la valeur théorique $Z_{t}=V_{c} / K \widetilde{u_{*}}$

\section{Etude expérimentale}

Nous avons mesuré la distribution des concentrations suivant la verticale au milieu d'un canal vitré de section rectangulaire, à partir d'échantillons extraits par le tube de prélèvement identique au tube de pitot, placé en sens contraire de l'écoulement. Le tube a été relié à une pompe rotative et la pompe elle-même rattachée à un rhéostat avec lequel nous pouvions régler la vitesse de rotation et, par conséquent, la vitesse d'aspiration de la suspension. Grâce à ce système de circuit, nous avons prélevé des échantillons avec une vitesse égale à celle de l'écoulement, au point de prélèvement. Pour cela, il fallait connaître tout d'abord la distribution des vitesses et la section d'entrée du tube de prélèvement des échantillons. Si la vitesse locale moyenne de l'écoulement, à la distance $y$ du fond du canal, était $\bar{u}_{d}$, son débit local pénétrant dans la section du tube de prélèvement $s=\frac{\pi d^{2}}{4}$ serait :

$$
q_{l}=\bar{u}_{d} s=\frac{\pi d^{2}}{4} \cdot \bar{u}_{d}
$$

pour que la vitesse de prélèvement soit $\bar{u}_{d}$, il faut donc que le débit extrait $q_{e}=\frac{\Delta V}{\Delta t}$ soit égal au débit local $q_{l} ;$ c'est-à-dire que nous devons avoir :

$$
q_{l}=q_{e} \quad \text { ou } \quad \bar{u}_{d} s=\frac{\Delta V}{\Delta t}
$$

où $\Delta V$ représente la volume de l'échantillon prélevé pendant un intervalle de temps $\Delta t$. En effet, en changeant la vitesse de rotation de la pompe à l'aide du rheostat, nous avons pu ohtenir le débit souhaité. Il est clair que si la vitesse de prélèvement était supérieure à celle du courant local, la quantité de matériaux solides dans les échantillons prélevés, diminuerait, car la phase liquide, de faible densité $\left(\rho_{s} / \rho_{L} \sim 2,5\right)$, serait absorbée dans le tube plus vite que la phase solide.

Nous avons effectué 3 séries d'essais et pour chaque série, nous avons réalisé 3 expériences dont les condi- tions hydrauliques étaient quasiment semblables ; seules les concentrations de transport $C_{T m}$ variaient. La moyenne de 3 prélèvements en chaque point a été considérée comme la concentration massique moyenne locale $\bar{C}$. Le $1^{\text {er }}$ prélèvement a été effectué à partir de $y=y_{0}=2 \mathrm{~mm}$, du fond du canal, sauf pour la $1^{\grave{e} \text { re }}$ série, où le $1^{\text {er }}$ échantillonnage a été réalisé à partir de $y=y_{0}=0,5 \mathrm{~mm}$. En fait, pour éviter la discontinuité et ne pas tomber dans la zone de sous-couche visqueuse $\delta$, nous avons estimé que $y_{0}$ doit être supérieur ou égal à $\delta$, c'est-à-dire $y_{0} \geqslant \delta$. Pour la série $\mathrm{I}, y_{0} \cong$ $2,77 \delta$ et pour les séries II et III, $y_{0} \cong 11 \delta ; \delta=$ $0,182 \mathrm{~mm}$ en moyenne dans nos expériences.

\section{Résultats concernant la distribution des} concentrations

Les résultats expérimentaux ont été comparés avec l'étude théorique du problème (3), (4). Les valeurs mesurées $Z_{m}$ sont en général supérieures à celles théoriques $Z_{t}$, calculées par la relation $Z_{t}=V_{c} / K \tilde{u}_{*}$. La différence relative est comprise entre 19 et $42 \%$. L'analyse de l'ensemble de nos résultats montre que les courbes théoriques et expérimentales coïncident dans la zone proche de la paroi, mais au-delà de cette zone, elles s'écartent l'une de l'autre. Pour pouvoir expliquer cet écart, nous sommes tentés d'en chercher l'origine au sein des hypothèses introduites dans la théorie; en effet, parmi celle-ci non justifiée, on a : l'hypothèse d'égalité du coefficient de diffusion de la phase solide $D_{s}$ et de la phase liquide $D_{L}$; c'est-à-dire $D_{s}=$ $D_{L}$. Afin de connaître l'allure générale de la variation du $D_{s}$ et du $D_{L}$, nous les avons calculés par les relations (1) et (2). Ces résultats nous permettent d'affirmer que, d'une part, la variation de $D_{s+}$ avec $y / h$ est quasiment parabolique, comme c'est le cas pour $D_{L+}$, et d'autre part que $D_{L}$ est supérieur à $D_{s}$

\section{Conclusion et discussion}

A l'issue de l'ensemble de ces résultats, nous tirons les conclusions suivantes :

- La loi logarithmique des vitesses est valable pour des écoulements diphasiques liquide-solide à surface libre.

- La constante de Karman $K$ (ou $A$ ) n'est pas une véritable constante ; elle varie avec la concentration et la dimension des particules.

- La présence des particules solides dans l'écoulement influence l'intensité de turbulence.

- La présence de suspension provoque une survitesse dans la partie centrale de l'écoulement diphasique liquide-solide.

- La présence de grandes valeurs des concentrations près de la paroi provoque une rugosité artificielle mobile sur celle-ci, ce qui fait varier $B$ dans le profil logarithmique des vitesses.

- La loi théorique de répartition des concentrations relatives suivant la verticale est valable dans la zone près de la paroi où la loi logarithmique des vitesses est applicable. 
- Le coefficient de diffusion de la phase solide $D_{s}$ est plus petit que celui de la phase liquide $D_{L}$; nous devons donc avoir un facteur de correction $\beta$, tel que : $D_{s}=\beta D_{L} ; \beta$ étant toujours inférieur à l'unité. En effet, l'expression de $D_{L}$ est seulement valable dans la zone près de la paroi où la loi logarithmique des vitesses est applicable ; tandis que, dans la zone loin de la paroi, l'expérience montre qu'il faut prendre une autre loi logarithmique des vitesses, avec $K^{\prime}<K$.

- L'exposant $Z$ de l'équation théorique (3) doit être corrigé par le facteur $\beta$; on aura alors : $Z=V_{c} / \beta K \tilde{u}$.

\section{Références bibliographiques}

[1] FORTIER A. - Mécanique des suspensions. Masson et Cie - Paris (1967).

[2] VANONI V.A. - Transactions of the American Society of Civil Engineers, vol. III, n 2267 (1946).

[3] MEYER-PETER E. et MULLER R. - Association Internationale de Recherches Hydrauliques, $2^{\mathrm{e}}$ Congrès Stockholm (1948).

[4] EINSTEIN H.A. et NING-CHIEN. - University of California, série $\mathrm{n}^{\circ} 33$, issue $\mathrm{n}^{\circ} 2,(1955)$.

[5] GARDE R.J. et PAINTAL A.S. - La Houille Blanche, $n^{\circ} 6(1964)$.

[6] DURAND R. - Transport hydraulique des matériaux solides en conduite. La Houille Blanche, (1951).

[7] ABOURAYAN M. - L'écoulement turbulent d'une suspension dans un canal. Thèse de Docteur-ingénieur, Paris VI (1974).

[8] HASSANZADEH Y. - Etude de l'écoulement d'une suspension hydraulique dans un canal. Thèse de Docteuringénieur, Université Pierre et Marie Curie, Paris VI, (1978).

[9] O'BRIEN M.P. - Review of the theory of turbulent flow and its relation to sediment transportation. Transaction A.G.U., vol. 14 (1933).
[10] ROUSE H. - Fluid mecanics for hydraulic engineers, McGraw-Hill Book Co (1938)

\section{Notations}
Symbole

$A, B$

$A^{\prime}, B^{\prime}$

Coefficients de la loi logarithmique des vitesses (près de la paroi).

\section{$\frac{C}{C}$} Coefficients de la loi logarithmique des vitesses (loin de la paroi).

Coefficients de la loi des vitesses déficitaires. Concentration massique moyenne locale à la cote $y$

$\bar{C}_{0} \quad$ Concentration massique moyenne locale à la cote $y_{0}$

$C_{T m} \quad$ Concentration de transport massique.

$\bar{d}^{T m} \quad$ Diamètre moyen des particules solides.

$D_{s} \quad$ Coefficient de diffusion de la phase solide.

$D_{L} \quad$ Coefficient de diffusion de la phase liquide.

$g \quad$ Accélération de la pesanteur.

$K=2,3 / A \quad$ Constante de Karman (près de la paroi)

$K^{\prime}=2,3 / A^{\prime} \quad$ Constante de Karman (loin de la paroi).

$R e$

$R_{H} \quad$ Rayon hydraulique.

$\bar{u} \quad$ Vitesse locale moyenne à la cote $y$.

$\tilde{v}=Q / S \quad$ Vitesse débitante.

$v \quad$ Vitesse moyenne.

$U \quad$ Vitesse maximum.

$V_{c} \quad$ Vitesse limite de chute.

$u_{*} \quad$ Vitesse de frottement local.

$\tilde{u}_{*} \quad$ Vitesse de frottement moyenne sur les parois.

$\rho_{L}, \rho_{s} \quad$ Masse volumique respective du liquide et solide.

$\nu \quad$ Viscosité cinématique du liquide.

$\lambda \quad$ Coefficient de perte de charge.

$\beta \quad$ Coefficient de correction. 